\title{
Near-field imaging of light propagation in photonic crystal waveguides: Explicit role of Bloch harmonics
}

Bozhevolnyi, Sergey I.; Volkov, V.S.; Søndergaard, Thomas; Boltasseva, Alexandra; Borel, Peter Ingo; Kristensen, Martin

Published in:

Physical Review B Condensed Matter

Link to article, DOI:

10.1103/PhysRevB.66.235204

Publication date:

2002

Document Version

Publisher's PDF, also known as Version of record

Link back to DTU Orbit

Citation (APA):

Bozhevolnyi, S. I., Volkov, V. S., Søndergaard, T., Boltasseva, A., Borel, P. I., \& Kristensen, M. (2002). Nearfield imaging of light propagation in photonic crystal waveguides: Explicit role of Bloch harmonics. Physical Review B Condensed Matter, 66(23), 235204. https://doi.org/10.1103/PhysRevB.66.235204

\section{General rights}

Copyright and moral rights for the publications made accessible in the public portal are retained by the authors and/or other copyright owners and it is a condition of accessing publications that users recognise and abide by the legal requirements associated with these rights.

- Users may download and print one copy of any publication from the public portal for the purpose of private study or research.

- You may not further distribute the material or use it for any profit-making activity or commercial gain

- You may freely distribute the URL identifying the publication in the public portal 


\title{
Near-field imaging of light propagation in photonic crystal waveguides: Explicit role of Bloch harmonics
}

\author{
Sergey I. Bozhevolnyi, ${ }^{1, *}$ Valentyn S. Volkov, ${ }^{1}$ Thomas Søndergaard, ${ }^{2}$ Alexandra Boltasseva, ${ }^{3}$ \\ Peter I. Borel, ${ }^{3}$ and Martin Kristensen ${ }^{3}$ \\ ${ }^{1}$ Institute of Physics, Aalborg University, Pontoppidanstroede 103, DK-9220 Aalborg Øst, Denmark \\ ${ }^{2}$ Micro Managed Photons A/S, COM, Building 345v, DK-2800 Kongens Lyngby, Denmark \\ ${ }^{3}$ COM, Technical University of Denmark, Building 345v, DK-2800 Kongens Lyngby, Denmark
}

(Received 30 July 2002; published 10 December 2002)

\begin{abstract}
We employ a collection scanning near-field optical microscope (SNOM) to image the propagation of light at telecommunication wavelengths along straight and bent regions of silicon-on-insulator photonic crystal waveguides (PCWs) formed by removing a single row of holes in the triangular 410-nm-period lattice along the $\Gamma M$ direction of the irreducible Brillouin zone. We obtain high quality SNOM images of PCWs excited in the wavelength range of 1520-1570 nm, which indicate good PCW mode confinement and low propagation loss. Using averaged cross sections of the intensity distributions before and after PCW bends, bend loss is evaluated and found to noticeably increase with the increase of the light wavelength from $\sim 1 \mathrm{~dB}$ at $1520 \mathrm{~nm}$ to $\sim 6 \mathrm{~dB}$ at $1570 \mathrm{~nm}$. We analyze light intensity variations along PCWs measured with the SNOM at different distances from the sample surface. Considering the interference between a quasihomogeneous background field and Bloch harmonics of the PCW mode, we account for spatial frequency spectra of the intensity variations and determine the propagation constant of the PCW mode at $1520 \mathrm{~nm}$. The possibilities and limitations of SNOM imaging for the characterization of PCWs are discussed.
\end{abstract}

DOI: $10.1103 /$ PhysRevB.66.235204

PACS number(s): 42.70.Qs, 07.79.Fc, 42.25.-p

\section{INTRODUCTION}

Interference in multiple scattering of light occurring in a medium with periodic modulation of the refractive index can lead, within a certain wavelength range, to light reflection from and inhibition of light propagation inside such a medium, which is conventionally called a photonic crystal (PC). ${ }^{1}$ This (photonic band-gap) effect opens a way to control the flow of light on a wavelength scale by introducing various defects, e.g., channels and cavities, in PCs. ${ }^{1,2}$ Twodimensional (2D) PCs combined with the structures exhibiting light confinement in the third dimension, e.g., planar waveguides, have been intensively investigated during recent years with the purpose of realization of highly integrated photonic circuits. ${ }^{2,3}$ Various configurations of PC waveguides (PCWs) formed by straight- and bent-line defects in 2D PCs have been experimentally studied, aimed at demonstrating low-loss propagation (and bending) of light, and many important issues have been clarified. ${ }^{4-13}$ It should be noted though that most of the experimental methods used so far are of an indirect nature. This limits the amount of accessible information and it may even compromise the validity of the conclusions reached.

Visualization of light propagation by imaging of the PCW surface with a camera appeared to be useful only with accidental ${ }^{4}$ or engineered ${ }^{5}$ defects present in the structure, or with light being intentionally coupled to leaky modes. ${ }^{6} \mathrm{Im}$ aging of the PCW output edge with a camera ${ }^{6}$ or a tapered fiber $^{7}$ was mainly employed to confirm that the light does go through a bent PCW in question. Relative measurements of the transmission spectra with and without a PCW (Refs. 8 and 9) (or with and without a bend ${ }^{10}$ ) with subsequent normalization were found to be generally reliable for loss mea- surements by calibrating away extrinsic effects, such as, e.g., laser-PCW-detector coupling. However the efficiency found in this way may exceed $100 \%,{ }^{8-10}$ indicating that accurate characterization of low-loss PCWs with this technique is somewhat problematic. Alternatively, one can make use of the Fabry-Perot resonance technique that was successfully applied for quantitative measurements of the propagation loss in straight PCWs (Ref. 11) and the modal reflection in bent PCWs. ${ }^{12}$ As far as PCW modal characteristics are concerned, conventional (far-field) imaging of the PCW surface has been recently found suitable for mapping the dispersion diagram of the leaky (and, thereby, lossy) PCW modes revealing Bloch wave components in the excited PCW modes. ${ }^{13}$ On the other hand, it is clear that a bound PCW mode having a propagation constant larger than that in air cannot be observed with far-field imaging techniques, because its field in air is evanescent, i.e., exponentially decaying over the distance of the order of light wavelength.

Mapping of evanescent fields is one of the main applications of a collection scanning near-field microscope (SNOM), whose fiber probe is used to pick up a tiny fraction of optical (e.g., evanescent) field near the sample surface and detect it as a function of scanning coordinates. Since the first demonstration of SNOM imaging of evanescent fields of waveguide modes ${ }^{14}$ the SNOM has been used in a number of studies concerned with waveguide modes in integrated optical components ${ }^{15}$ including waveguide characterization at telecommunication wavelengths. ${ }^{16-18}$ As far as photonic band-gap structures are concerned, the SNOM has been applied to study optical transmission through 2D PCs (Refs. 19 and 20) and to observe guiding of surface-plasmon polaritons along channels in a periodically corrugated metal surface. ${ }^{21}$ The SNOM imaging of photoluminescence in a 2D PC etched in a suspended InP membrane has also been 


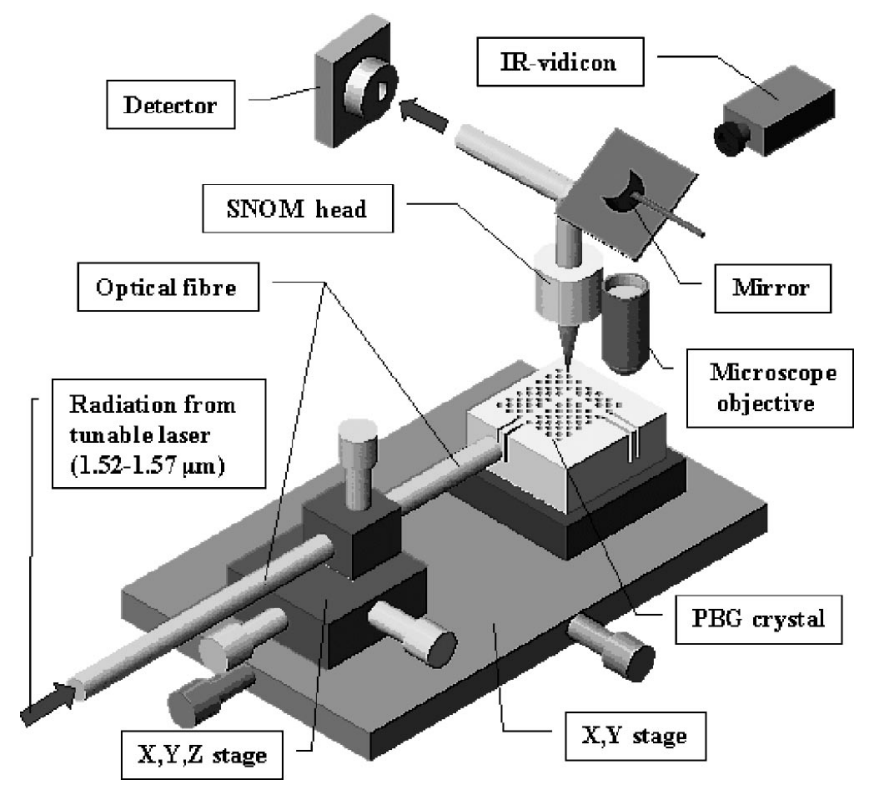

FIG. 1. Schematic layout of the experimental setup.

reported. ${ }^{22}$ However, the potential of this technique to map the intensity distribution of (bound) modes guided along PCWs has not yet been realized.

In this work, we apply a SNOM operating in collection mode to image the propagation of light at telecommunication wavelengths along straight and bent regions of silicon-oninsulator PCWs. A similar sample containing the PCWs with different lengths has been previously investigated with respect to the transmission loss in the wavelength interval of $1300-1600 \mathrm{~nm}$, and the propagation loss in straight parts of PCWs has been determined by linear regression. ${ }^{23}$ We obtain high quality SNOM images that allow us to directly evaluate the bend loss and characterize the PCW mode confinement. From the SNOM images we further determine the spatial frequencies in light intensity variations along the PCW that we interpret by considering the interference between a quasihomogeneous background field and Bloch components of the PCW mode. This allows us to estimate the propagation constant of the PCW mode at $1520 \mathrm{~nm}$. It should be stressed that the main purpose of this work is to explore the possibilities and limitations of SNOM imaging for the PCW characterization rather than to study a particular type of PCWs. The paper is organized as follows. In Sec. II the experimental arrangement and the sample used are described. Section III is devoted to the experimental results featuring the SNOM images obtained at different sample areas and their interpretation. The paper is terminated with our conclusions offered in Sec. IV.

\section{EXPERIMENTAL ARRANGEMENT}

The experimental setup consists of a collection SNOM with an uncoated fiber tip used as a probe ${ }^{24}$ and an arrangement for launching tunable (1520-1570 nm) TE-polarized (the electric field is parallel to the sample surface) radiation (power $\sim 1 \mathrm{~mW}$ ) into the sample waveguides by positioning a tapered lensed single-mode fiber (Fig. 1). The sample con-
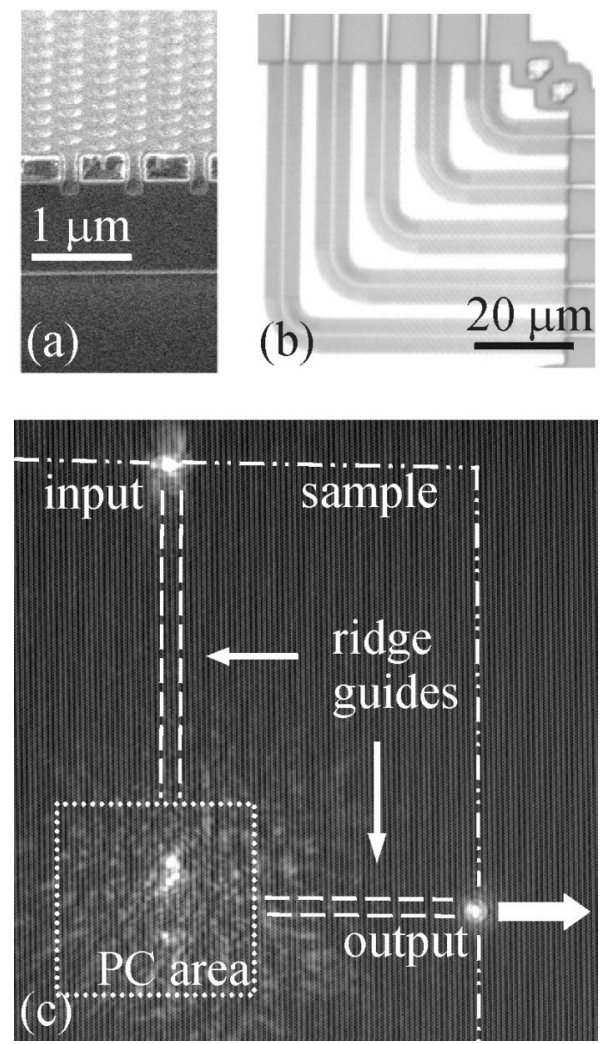

FIG. 2. (a) Scanning electron microscope picture showing a cross-sectional view of a reference sample, which has been cleaved through the photonic crystal (PC) area. (b) Optical microscope image showing a top view of the central part of the sample, which contains bent PC waveguides. (c) Far-field image taken with a camera positioned over the top of the sample, with the radiation at 1520 $\mathrm{nm}$ being coupled from a cleaved fiber (not seen) into the input ridge waveguide leading to the PC area. The wide arrow points in the propagation direction of radiation coupled out of the output ridge waveguide.

taining PCWs consisted of a perforated $\mathrm{SiO}_{2} / \mathrm{Si} / \mathrm{SiO}_{2}$ trilayer film (cladding/core/buffer thickness $\sim 0.1 / 0.3 / 1 \mu \mathrm{m}$ ) formed on a Si substrate. ${ }^{23}$ Holes were arranged in a triangular array (period $\Lambda \cong 410 \mathrm{~nm}$, hole diameter $\cong 200 \mathrm{~nm}$ ) and single rows of missing holes defined the PCWs along $\Gamma M$ direction of the irreducible Brillouin zone of the lattice. ${ }^{1}$ E-beam lithography was employed to produce the hole pattern in a resist layer deposited on a silicon-on-insulator wafer. The patterned resist served as a mask in the process of reactive ion etching (RIE), resulting in the corresponding pattern of holes formed in the Si layer. After removal of the resist, the pattern was further transferred onto the $\mathrm{SiO}_{2}$ layer by RIE, using the patterned $\mathrm{Si}$ layer as a mask. Finally, a $\mathrm{SiO}_{2}$ cladding layer was grown on the Si-layer surface by thermal oxidation completing the sample fabrication [Fig. 2(a)]. The choice of PCW parameters was based on the simulations carried out with a plane-wave expansion approach ${ }^{25}$ predicting for similar sample leakage-free guided TE modes near the wavelength of $1450 \mathrm{~nm}^{23}$ The sample contained a central PC square area with five PC regions forming bent PCWs of different lengths connected to tapered access ridge waveguides outside the PC area [Fig. 2(b)]. Each waveguide 


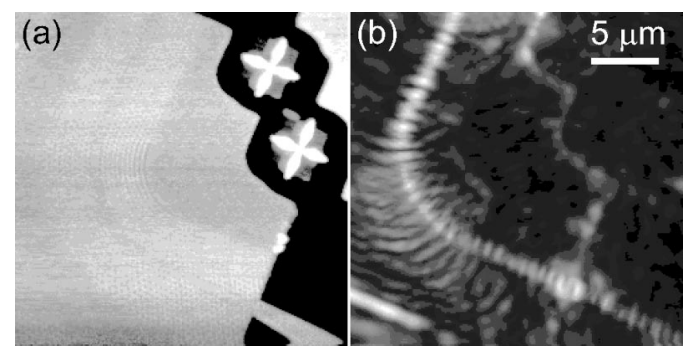

FIG. 3. Gray-scale (a) topographical and near-field optical images $\left(22.5 \times 22.5 \mu \mathrm{m}^{2}\right)$ obtained with the shortest PCW [Fig. 2(b)] at the wavelength $\lambda \cong 1520 \mathrm{~nm}$. One can distinguish the line of missing holes that defines the PCW and clearly see the output ridge waveguide in the topographical image whose depth is $1.9 \mu \mathrm{m}$.

had two straight sections connected by a $90^{\circ}$ bend obtained by an adiabatic rotation (with the curvature radius of $7 \mu \mathrm{m}$ ) of the crystal lattice. Cleavage of the sample resulted in $\sim 150$ - $\mu \mathrm{m}$-long and $\sim 4-\mu \mathrm{m}$-wide (at the sample facets) ridge waveguides leading toward each side of the PC area.

The adjustment of the in-coupling fiber with respect to the access ridge waveguides was accomplished when monitoring the light propagation along the sample surface with the help of a far-field microscopic arrangement (Fig. 1). A typical image featured only input and output bright spots at the sample facets along with some weak spots near waveguide bends in the PC area [Fig. 2(c)]. These measurements indicated that the main part of propagation loss is associated with the bends in PCWs and that the loss increases with the wavelength as expected from the transmission measurements carried out previously. ${ }^{23}$ Following the fiber adjustment, the fiber-sample arrangement is moved under the SNOM scanning head, and the optical-field distribution near the sample surface is probed with an uncoated sharp fiber tip, whose position is controlled by the SNOM head (Fig. 1). The tip can be scanned along the sample surface at a constant distance (of a few nanometers) maintained by shear force feedback or along a plane, which is approximately parallel to the surface plane and separated from it by a given distance. The near-field radiation scattered by the fiber tip is partially collected by the fiber itself and propagates in the form of fiber modes towards the other end of the fiber, whereupon it is detected by a femtowatt InGaAs photoreceiver. Finally, it should be noted that the images presented here [Figs. 2(c) and $3-8$ ] are oriented in the same way with respect to the direction of light propagating in the PCWs.

\section{EXPERIMENTAL RESULTS}

All five PCWs were in turn excited at different wavelengths ranging from $1520 \mathrm{~nm}$ to $1570 \mathrm{~nm}$ and imaged with the SNOM. The topographical and near-field optical images obtained for the shortest PCW at the wavelength of $1520 \mathrm{~nm}$ are shown in Fig. 3. On the topographical image, one can recognize the pattern of holes that forms the PCW following by the output ridge waveguide and even distinguish individual holes albeit only in the output PCW region [Fig. 3(a)]. In general, the visibility of holes on the topographical images was found to vary even within the same image. This feature is an experimental artifact related to the lattice orientation and the scan direction, the scan step, and feedback parameters that were not optimized with respect to the topographical imaging. The near-field optical image [Fig. 3(b)] exhibits several features appearing also on the images of other PCWs and/or at other wavelengths. The light propagation along the bent PC waveguide and further in the access ridge waveguide is clearly seen, as well as the light scattering at the junction between the PCW and ridge waveguide. The optical signal related to the light propagation in the PCW decreases noticeably after the bend. The radiation bend loss is visualized on the image appearing in the form of light scattering out of the bend in the forward direction that illuminates the edge of the neighbor $\mathrm{PC}$ region. Note that the light scattering at the junction between the PCW and ridge waveguide and at the edge of the PC region (near the bend) results in scattered field components propagating (in air) away from the sample surface. These propagating components are detected with the SNOM fiber tip much more efficiently than the evanescent field components, ${ }^{26}$ e.g., those associated with the PCW mode. One should therefore be very careful when judging the strength of scattering processes from SNOM images: scattering into air gives considerably stronger signals than scattering in the surface plane or scattering into the substrate. Finally, the light propagating along the PCW is well confined to the line of missing holes showing a somewhat irregular periodic pattern in the propagation direction [Fig. 3(b)]. In the following, we shall take a closer look at light propagation in the bent and straight regions of the PCWs.

\section{A. Determination of the PCW bend loss}

The variations $(\sim 30 \%)$ of the detected signal along the PCW axis made the precise evaluation of the propagation loss in straight parts of PCWs rather difficult. Even for the longest PCW $(\sim 50 \mu \mathrm{m})$, the propagation loss could be at best estimated as being within $10 \%$ and somewhat larger for longer wavelengths. These observations are in agreement with the transmission measurements conducted with a similar sample, that resulted in the propagation loss of a few $\mathrm{dB} / \mathrm{mm}$ at $1520 \mathrm{~nm}$, increasing with the wavelength. ${ }^{23}$ Note that the bend loss could not have been determined from the transmission measurements, because the coupling loss contributes to the measured overall transmission loss in the same manner. Using the SNOM images obtained at different wavelengths (Fig. 4), we have directly determined the bend loss by averaging the intensity distributions (across the PCW) over the PCW length of $\sim 5 \mu \mathrm{m}$ before and after the bend. The bend loss was found to increase with the wavelength from the value of $\sim 1 \mathrm{~dB}$ at the wavelength of $1520 \mathrm{~nm}$ to that of $\sim 6 \mathrm{~dB}$ at $1570 \mathrm{~nm}$. These results are consistent with our far-field observations mentioned above and with the fact that the propagation loss is expected to increase notably for the light wavelengths considerably different from those ensuring the leakage-free guidance. ${ }^{25}$

The SNOM images allow one to determine the bend loss rather accurately, i.e., within a few percent, for a given PCW and particular adjustment of the in-coupling tapered fiber. However, the determined bend loss was found to noticeably 
vary not only for different PCWs but also for different adjustments of the fiber with respect to the same PCW, especially with respect to the fiber displacement perpendicular to the surface plane. A closer look at SNOM images revealed that the light intensity distribution across a PCW became broader and the bend loss increased when the in-coupling fiber was moved deeper in the sample. The typical SNOM images recorded in this configuration at the wavelength of $1520 \mathrm{~nm}$ are shown in Fig. 5. It is seen that, in the straight PCW region preceding the bend, the intensity distribution is rather broad and exhibits two maxima on both sides of the PCW. At the same time, after the bend, it is confined to the PCW region, which is quite similar to that obtained with another fiber adjustment [cf. Figs 4(b) and 5(b)].

A possible explanation might be related to the mode structure of the ridge access waveguides. We considered a planar waveguide formed by the trilayer film on a Si substrate (see Sec. II), and found that it supports two TE modes. One mode is concentrated in the $\sim 0.3-\mu \mathrm{m}$-thin Si layer and in its immediate vicinity, whereas another one is propagating mainly within the $\sim 1-\mu$ m-thick $\mathrm{SiO}_{2}$ layer. We expect that similar (in depth) modes are supported also by the ridge waveguides and that these modes can be excited separately or simultaneously depending on the in-coupling fiber position with respect to the sample surface. The etched holes being relatively shallow [Fig. 2(a)] affect strongly the first mode and only slightly the second one, a circumstance that can account for the aforementioned observations. The PCW bend plays, in such a case, the role of a mode stripper, effectively filtering the second mode out of the PCW. Hereafter we consider the measurements conducted with the appropriate fiber adjustments judged from the SNOM images.

The fluctuations in the bend loss measured with different waveguides and fiber adjustments complicate the bend loss evaluation for a particular PCW design. We should also mention another complication, which is inherent to the usage of SNOM imaging, viz., high-resolution and large scale SNOM images take a long time to acquire. In our case, a SNOM image of $40 \times 40 \mu \mathrm{m}^{2}$ with $256 \times 256$ points took $\sim 1.5 \mathrm{~h}$ of recording, because the detection of weak $(\sim 1 \mathrm{pW})$ and noisy optical signals requires a long integration time $(\sim 0.1 \mathrm{~s})$. This circumstance makes the usage of data statistical treatment rather problematic. We have made most measurements at the wavelength of $1520 \mathrm{~nm}$, and the bend loss of $\sim 1 \mathrm{~dB}$ was obtained under a proper fiber adjustment for the longest and middle PCWs that featured the light propagation with less scattering. The tendency of bend loss increase with the wavelength (Fig. 4) was also quite reproducible, but other values of bend loss, e.g., that of $6 \mathrm{~dB}$ at $1570 \mathrm{~nm}$, can be given only with $50 \%$ accuracy.

\section{B. Scattering out of PCW bends}

The radiation scattered out of the PCW bend is seen to propagate along the sample surface and diverge from the bend, forming weak but noticeable concentric fringes (Figs. 4 and 5). Similar fringes have been previously reported in the SNOM study of semiconductor waveguide structures by Bourzeix et al. ${ }^{16}$ and attributed to the Tien effect. We believe that this and similar phenomena observed in SNOM studies of waveguides ${ }^{16,18}$ and accounted for previously with the Tien effect are in fact interference phenomena caused by the SNOM detection of weak optical fields forming a quasihomogeneous background. Let us recall that, in the detection process of a collection SNOM, a fiber probe picks up planewave components of the detected field with the efficiency that depends on their wave-vector projection on the surface plane. ${ }^{26}$ All these plane-wave components contribute linearly (though differently) to the amplitude of the fiber mode(s) propagating toward a detector. It should also be borne in mind that the efficiency decreases drastically with the increase of the wave-vector projection magnitude. ${ }^{27}$ It is therefore reasonable to expect that, in the SNOM imaging of waveguide modes, especially those with large effective indexes, even weak scattered optical fields caused by material inhomogeneities and fabrication defects can contribute to the detected signal. Note that such a contribution is coherent and strongest from the optical fields with small wave-vector projections. One should therefore expect to observe on the SNOM image an interference pattern superimposed on the image formed by a waveguide mode. Moreover, this interference pattern being somewhat irregular due to different contributing plane-wave components should exhibit, on average, the interference fringes corresponding to the optical field with zero wave-vector projection (propagating away from the surface), i.e., to the homogeneous coherent background.

The above explanation is consistent with the reported observations of quasiperiodic intensity modulations along the propagation direction in SNOM images of waveguides. ${ }^{16,18}$ Thus, the average oscillation period of $0.44 \pm 0.10 \mu \mathrm{m}$ for semiconductor waveguides matches their effective index $\left(\lambda / n_{\text {eff }}=0.48 \pm 0.04 \mu \mathrm{m}\right)$, and the period of $\approx 1 \mu \mathrm{m}$ observed at $\lambda=1.55 \mu \mathrm{m}$ for polymer and glass waveguides is consistent with the expected effective index $n_{\text {eff }} \sim 1.5 .{ }^{16}$ One

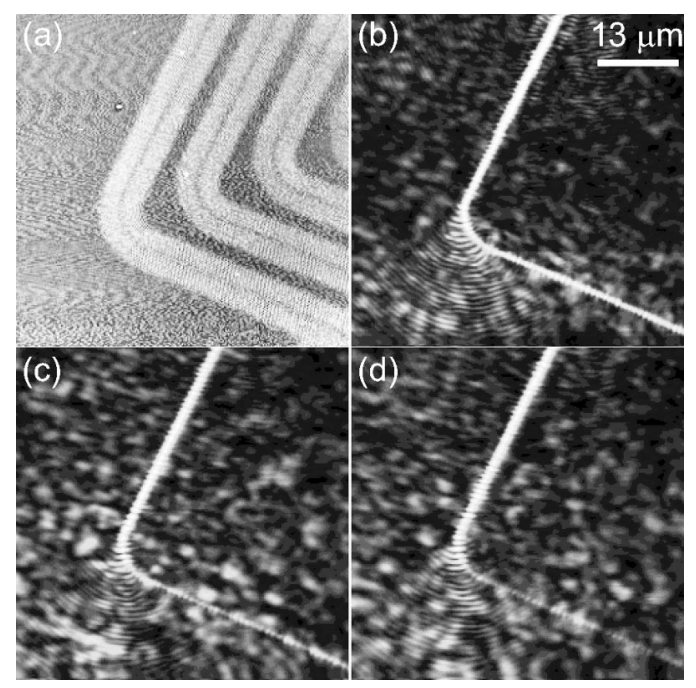

FIG. 4. Gray-scale (a) topographical and (b)-(d) near-field optical images $\left(52.5 \times 52.5 \mu \mathrm{m}^{2}\right)$ obtained with the longest PCW [Fig. 2(b)] at the wavelengths $\lambda \cong$ (b) 1520 , (c) 1550 , and (d) 1570 $\mathrm{nm}$. Appearance of weak optical signals is artificially enhanced in order to improve the visibility of concentric fringes originating at the PCW bend. The depth of the topographical image is $0.51 \mu \mathrm{m}$. 


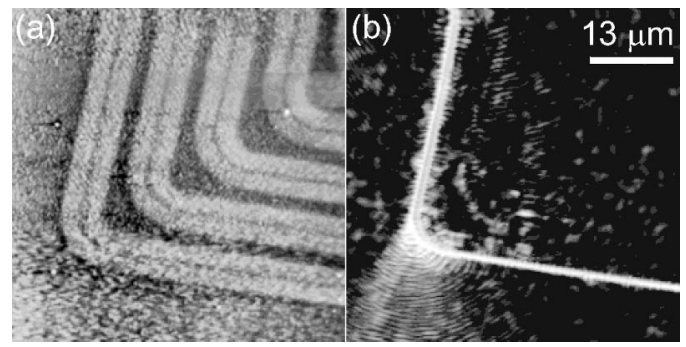

FIG. 5. Gray-scale (a) topographical and near-field optical images $\left(52.5 \times 52.5 \mu \mathrm{m}^{2}\right)$ obtained at the wavelength $\lambda \cong 1520 \mathrm{~nm}$ with the in-coupling fiber displaced perpendicular to the sample surface deeper inside the sample (as compared to the coupling configuration used to record the images shown in Fig. 4). The bend loss measured in this case is $\sim 3 \mathrm{~dB}$. The depth of the topographical image is $0.17 \mu \mathrm{m}$.

can also notice that the period of the phase distribution measured for the guided mode is close to that of the (irregular) stripe pattern in the corresponding intensity distribution. ${ }^{18}$ In our case, the presence of scattered optical fields propagating away from the surface was directly revealed by recording the optical images at different tip-surface distances (Fig. 6). It is seen that, with the increase of the tip-surface distance up to $500 \mathrm{~nm}$, the average optical signal rapidly decreases, the images become blurred, i.e., lose their high spatial frequencies, and the concentric fringes associated with the radiation diverging from the bend disappear. For even larger distances, the images change very slowly having the same signal level of $\sim 10 \%$ from the maximum signal recorded at the surface [cf. Figs. 6(d) and 6(f)]. These features indicate that the concentric fringes are associated with evanescent waves and that the propagating field components with small wave-vector projections do contribute to the detected signal, since only low spatial frequencies are seen at large distances.

A closer look at the concentric fringes revealed that their structure is somewhat irregular, showing features associated with the presence of phase singularities. ${ }^{28}$ This is only to be expected since the background contribution consists of many propagating field components with small but nonzero wavevector projections on the surface plane. For this reason, different radial cross sections of the concentric fringes observed at the wavelength of $1520 \mathrm{~nm}$ result in slightly different periods centered near $850 \mathrm{~nm}$ (Fig. 7). Note that different periods can also originate from a finite length of the cross sections $(\sim 20 \mu \mathrm{m}$ in this case). We evaluated the effective index of the TE mode concentrated in the Si layer of a planar waveguide: $n_{\mathrm{eff}} \approx 1.72$. For this value, the average period of interference fringes is expected to be equal to $\lambda / n_{\text {eff }}$ $\approx 880 \mathrm{~nm}$. Taking into account that the thickness $(\sim 300 \mathrm{~nm})$ of the core silicon layer of our sample influences strongly the effective mode index (e.g., for the thickness of $310 \mathrm{~nm}, n_{\text {eff }}$ $\approx 1.79$ and $\lambda / n_{\text {eff }} \approx 850 \mathrm{~nm}$ ), we consider the agreement as being reasonably good.

\section{Propagation in straight PCWs}

The near-field optical images obtained with high resolution at different light wavelengths for a straight region of the
PCW are shown in Fig. 8. It is seen that the recorded intensity distributions are well confined (to the line of missing holes defining the PCW) exhibiting wavelength-dependent intensity variations along the propagation direction. The signal cross sections made at the intensity maxima are very

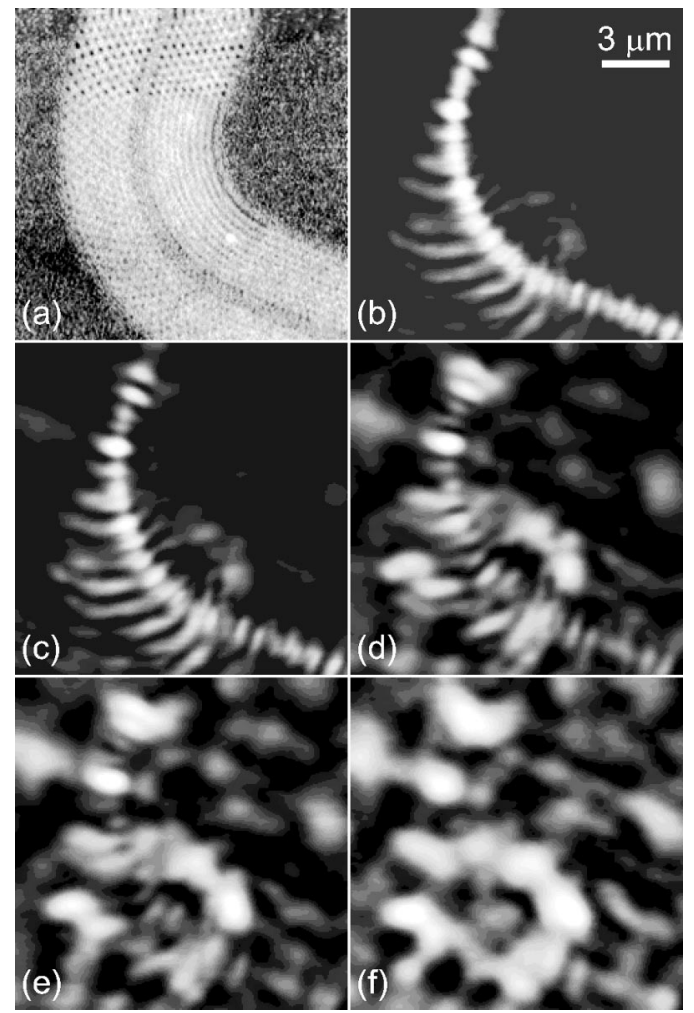

FIG. 6. Gray-scale (a) topographical and (b)-(f) near-field optical images $\left(15 \times 15 \mu \mathrm{m}^{2}\right)$ obtained at the wavelength $\lambda \cong 1520 \mathrm{~nm}$ at different tip-surface distances: (b) with shear force feedback (a few nanometers), (c) 100, (d) 300, (e) 500, and (f) $700 \mathrm{~nm}$. The maximum signal level is different for different images: (b) 1, (c) 0.46 , (d) 0.16 , (e) 0.13 , and (f) 0.11 . The line of missing holes defines the PCW and some of the holes are clearly seen on the topographical image whose depth is $0.11 \mu \mathrm{m}$.

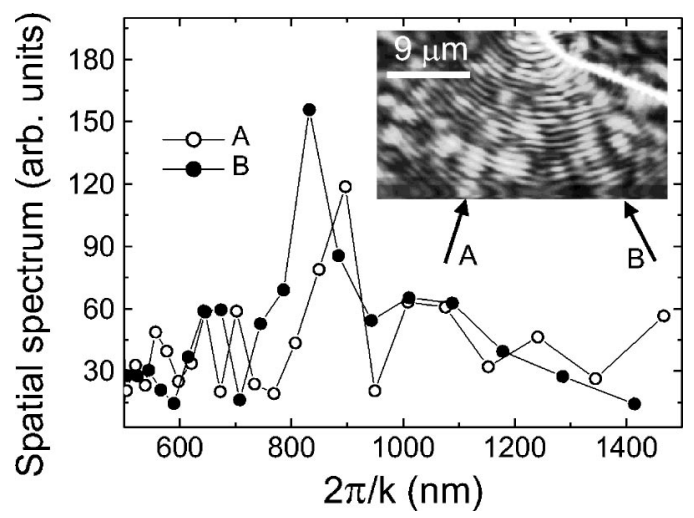

FIG. 7. Spectra of spatial frequencies of the cross sections of the concentric fringes (along the lines marked with arrows) that are imaged at the wavelength $\lambda \cong 1520 \mathrm{~nm}$ and shown in the inset. The size of the inset optical image is $32 \times 19 \mu \mathrm{m}^{2}$. 
close to the Gaussian distributions in shape with the full width at half maximum increasing from $\sim 740 \mathrm{~nm}$ to $800 \mathrm{~nm}$ with the increase of the light wavelength (Fig. 9). One can also notice that the signal does not go to zero outside the PCW indicating the presence of a homogeneous background at the level of $\sim 10 \%$ compared to the maximum signal. This circumstance should be borne in mind when accounting for the intensity variations along the PCW. It should be stressed that a 10\%-high level of signal background does not mean that the scattered field intensity on average is only 10 times smaller than the PCW mode intensity. The scattered field intensity is in fact considerably smaller than that, because the detection efficiency of fields with low spatial frequencies is much higher than that of the evanescent PCW mode. ${ }^{26,27}$

The intensity distributions measured along the PCW (Fig. 8) indicate that there are several spatial frequencies involved, with the main contribution coming from the frequencies centered near the period of $1 \mu \mathrm{m}$. In order to deduce the PCW characteristics from the corresponding SNOM images we start by writing the mode field distribution inside the PCW: ${ }^{1,13}$

$$
E(x, y, z)=\sum_{m} u_{m}(y, z) \exp [i(\beta+m K) x] ; \quad K=\frac{2 \pi}{\Lambda},
$$

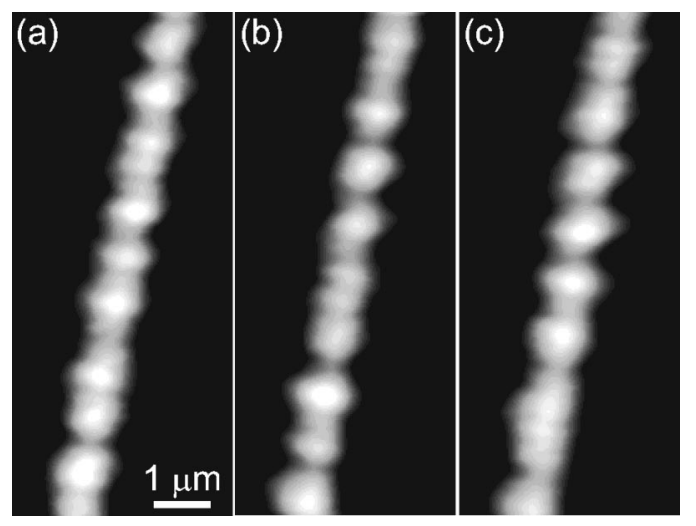

FIG. 8. Gray-scale near-field optical images $\left(4 \times 9 \mu \mathrm{m}^{2}\right)$ obtained with a straight region of PCW at the wavelengths $\lambda \cong(\mathrm{a})$ 1520, (b) 1550, and (c) $1570 \mathrm{~nm}$.

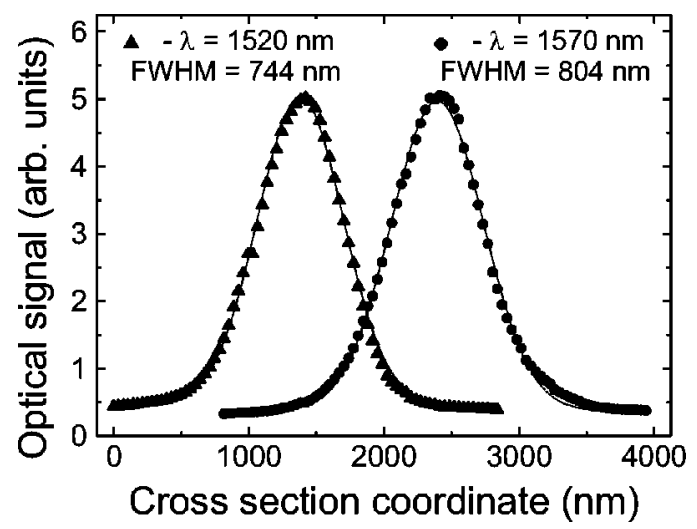

FIG. 9. Cross sections of the near-field optical images shown in Figs. 8(a) and 8(c) perpendicular to the PCW axis. Solid lines indicate the Gaussian fits to the experimental data.

$$
m=0, \pm 1, \pm 2, \ldots
$$

where $E(x, y, z)$ is the main electric-field component of the PCW mode propagating along the $x$ axis of the PCW and having the propagation constant $\beta(\beta \leqslant 0.5 \mathrm{~K})$, and $\Lambda$ is the PC lattice constant. The field, that is probed by the SNOM above the sample surface and along the PCW axis $(y=0)$, can then be approximated as follows:

$$
\begin{aligned}
E_{d}(x, z)= & B+\sum_{m} u_{m}^{0} \exp \left[-z \sqrt{(\beta+m K)^{2}-k_{0}^{2}}\right] \\
& \times \exp [i(\beta+m K) x]
\end{aligned}
$$

where the $z$ axis is perpendicular to the sample surface $(z$ $=0)$ pointing to the air side, $B$ represents a homogeneous background (see the discussion in the previous section), $k_{0}$ $=2 \pi / \lambda$ is the wave number in air, and we assumed that all Bloch components are evanescent. Indeed, for the considered situation $(\Lambda \cong 410 \mathrm{~nm}, \lambda \cong 1520 \mathrm{~nm})$, the PCW mode propagation constant is expected to be in the range of $0.4-0.5 \mathrm{~K}^{25}$ implying that the propagation constants of all Bloch components are larger than the wave number in air.

Each of the field components described in Eq. (2) contribute to the fiber mode amplitude (which is actually detected), but the coupling efficiency decreases drastically with the increase of the wave-vector surface projection $|\beta+m K|{ }^{26,27}$ One reason is that the effective detecting center of a SNOM probe fiber is situated at some distance inside the fiber (up to $500 \mathrm{~nm}){ }^{29}$ so that the evanescent fields are always probed at a nonzero distance from the surface. Keeping only the leading terms, the signal detected (along the PCW) at the tipsurface distance $z$ reads

$$
\begin{aligned}
S(x, z) \propto & C(z)+2 h(0) B h(\beta) u_{0}^{0} \exp \left(-z / d_{0}\right) \cos (\beta x) \\
& +2 h(0) B h(K-\beta) u_{-1}^{0} \exp \left(-z / d_{1}\right) \cos [(K-\beta) x] \\
& +2 h(\beta) u_{0}^{0} h(K-\beta) u_{-1}^{0} \exp \left(-z / d_{2}\right) \cos (K x), \quad(3)
\end{aligned}
$$

where $C(z)$ denotes the level of signal background, whose distance dependence is due to the averaged intensity of the evanescent waves, $h(k)$ is the detection efficiency of the field component having the magnitude $k$ of wave-vector projection on the surface plane, and the penetration depths are given by [Eq. (2)]

$$
\begin{gathered}
d_{0}=\left(\beta^{2}-k_{0}^{2}\right)^{-0.5}, \quad d_{1}=\left[(K-\beta)^{2}-k_{0}^{2}\right]^{-0.5}, \\
d_{2}=\frac{d_{0} d_{1}}{d_{0}+d_{1}} .
\end{gathered}
$$

It is expected that $h(0) \gg h(\beta)>h(K-\beta)$ for the aforementioned reasons and, thereby, the signal harmonics having spatial frequencies $\beta$ and $K-\beta$ would dominate the spectrum [Eq. (3)].

We investigated in detail the light propagation in the PCW at the wavelength of $1520 \mathrm{~nm}$. The typical spectrum of the optical signal variations along the PCW measured at the surface (using shear force feedback) is shown in Fig. 10 featuring three peaks in agreement with the arguments above. The 


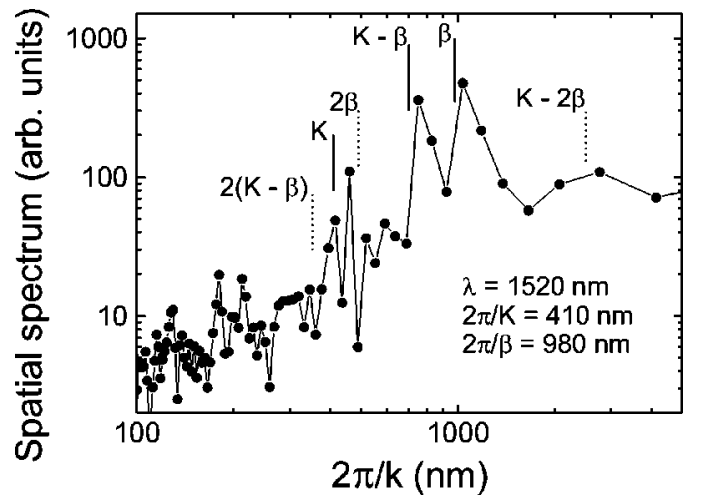

FIG. 10. Typical spatial spectrum of the optical image obtained with a straight region of PCW at the wavelength $\lambda \cong 1520 \mathrm{~nm}$. Positions of the main peaks fitted to the experimental data are indicated with solid lines. Dotted lines indicate combination frequencies that may appear due to the propagating backward PCW mode and/or nonlinear detection with a photoreceiver.

lattice constant of $410 \mathrm{~nm}$ of our PC was obtained from images made by a calibrated scanning electron microscope and corroborated with SNOM topographical images [e.g., similar to Fig. 6(a)]. The PCW mode propagation constant $\beta$ was fitted to the experimental spectrum so that the frequency $K-\beta$ would also fit the corresponding peak as shown in Fig. 10. One can further see that the spectrum structure, being well defined at low spatial frequencies, is more complicated around the frequency $K$ of the lattice constant. We think that such a structure might be related to the PCW mode reflected by the PCW-ridge waveguide interface and/or the output edge of the sample. The contribution to the detected signal due to the propagating backward PCW mode can be expressed similarly to Eq. (3),

$$
\begin{aligned}
S_{r}(x, z) \propto 2 \alpha\left[h(\beta) u_{0}^{0}\right]^{2} \exp \left(-2 z / d_{0}\right) \cos (2 \beta x) \\
\quad+2 \alpha\left[h(K-\beta) u_{-1}^{0}\right]^{2} \exp \left(-2 z / d_{1}\right) \cos [2(K-\beta) x] \\
\quad+4 \alpha h(\beta) u_{0}^{0} h(K-\beta) u_{-1}^{0} \exp \left(-z / d_{2}\right) \\
\quad \times \cos [(K-2 \beta) x],
\end{aligned}
$$

where $\alpha \ll 1$ is the amplitude reflection coefficient. It is seen that all three terms are of the same order of magnitude and smaller than the last term in Eq. (3) by the value of $\alpha$. Strictly speaking, these combination frequencies might also appear if the response of the photoreceiver used is not exactly linear with respect to the incident light power. We believe that the identification of these peaks and their origin is possible but requires additional and more detailed investigations. Here one should bear in mind the sources of uncertainty in the measured spectra mentioned in the previous section. In the following, we continue the consideration of the main peaks described by Eq. (3).

The modification of the optical images of a straight PCW region when increasing the tip-surface distance is similar to that reported above for the images of the PCW bend (Fig. 6). Averaging small details out (with the distance increase) is directly related to the corresponding changes in spatial frequency spectra that lose their high spatial frequencies. The

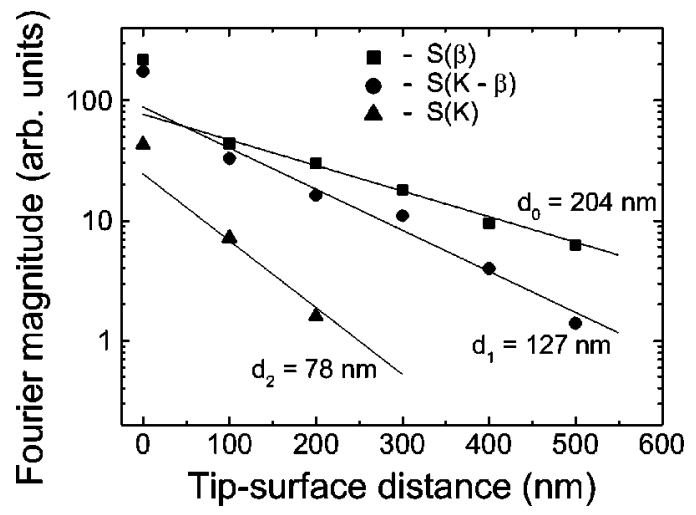

FIG. 11. Dependencies of the main spectral peaks shown in Fig. 10 on the tip-surface distance measured (symbols) and fitted (solid lines) according to their spatial frequencies.

expected distance dependencies of the three main spectral peaks are given by Eq. (3), and can be used to validate the above interpretation of the spatial spectrum (Fig. 10). The experimental data from the peak amplitudes are shown in Fig. 11 together with the exponential dependencies $A_{i} \exp$ $\left(-z / d_{i}\right)$ calculated from Eq. (4) with the spatial frequencies, which were found previously (Fig. 10). The prefactors $A_{i}$ of these dependencies were fitted disregarding the values measured at the surface, because the separation of the sample surface and the scanning planes (at distances $z>0$ ) is not well defined. In general, these planes are parallel only to the extent that the SNOM head axis is perpendicular to the sample surface. The detection efficiency of the fiber for these two configurations might be also somewhat different, e.g., due to the fiber bending caused by shear forces. With this in mind, we find the agreement between calculated and measured slopes as being rather good.

\section{CONCLUSIONS}

In this paper, using the SNOM in collection mode, we have imaged the propagation of light at telecommunication wavelengths along straight and bent regions of silicon-oninsulator PCWs formed by removing a single row of holes in the triangular 410-nm-period lattice. High quality SNOM images of PCWs excited in the wavelength range of 1520$1570 \mathrm{~nm}$ have been obtained and analyzed to determine the PCW characteristics. Thus, the bend loss has been directly evaluated using averaged cross sections of the intensity distributions before and after PCW bends. We have analyzed light intensity variations along $\mathrm{PCW}$ s measured with the SNOM at different distances from the sample surface and related these variations to Bloch components of the $\mathrm{PCW}$ mode. Using a phenomenological description of the SNOM imaging and assuming the presence of a quasihomogeneous background field, we have identified in spatial frequency spectra of the intensity variations the corresponding Bloch harmonics and determined the propagation constant of the PCW mode at $1520 \mathrm{~nm}$. 
The issues discussed in this work have implications for the SNOM characterization of any PCW and, at least, some types of conventional waveguide structures. Thus, we have demonstrated the importance of careful adjustment of the in-coupling fiber with respect to the access waveguides for the bend loss evaluation and PCW mode characterization. We have observed concentric fringes formed by the radiation scattered out of the PCW bend, which are similar to those reported in the SNOM study of semiconductor waveguide structures by Bourzeix et al. ${ }^{16}$ and attributed to the Tien effect. We have explained this phenomenon by interference between the contributions in the detected signal from the waveguide mode and a quasihomogeneous coherent background. This explanation was found to be consistent with the experimental results reported previously ${ }^{16,18}$ and those of our work. The presence of such a background might be considered as a drawback intrinsic for a particular configuration (of a PCW, access waveguide, coupling fiber, etc.) because it jeopardizes the accuracy of determination of spatial frequencies. On the other hand, it increases the detected signal due to the mode field, so that the signal becomes proportional to the field amplitude (not intensity) and thereby sensitive to the field phase. For example, the field phase map was directly displayed in the form of aforementioned concentric fringes. This feature is especially important for the SNOM imaging of waveguide modes having large effective indexes, e.g., in semiconductor waveguides, and rather short penetration depths in air, circumstances that makes them difficult to ef- ficiently detect. Note that there do exist conventional SNOM interferometric techniques, ${ }^{29,30}$ though their operation is quite delicate and very sensitive to the environmental conditions (temperature, vibrations, etc.). Finally, it should be stressed that the measurements of the spectral modifications with the tip-surface distance are crucial for the interpretation of the signal variations along the PCW measured with any SNOM technique.

We believe that the results presented in this work can help in designing further, more detailed and accurate, SNOM investigations of PCWs and, in general, PC structures. The accurate PCW characterization is particularly important since rigorous calculations of the properties of PCW components are extremely difficult and time consuming. The SNOM imaging can be used not only to quantitatively characterize properties of fabricated PCWs (mode profiles and propagation constants including loss) and PC structures (evaluation of loss and identification of loss channels) and thereby optimize their performance, but also to unveil many intriguing scattering phenomena occurring in PCs.

\section{ACKNOWLEDGMENT}

This research is carried out in the framework of the European project, Photonics Integrated Components based on Crystal Optics, and partially supported by the Danish Technical Research Council, Contract No. 26-00-0329.
*Corresponding author. FAX: (+45) 98156502. Email address: sergey@physics.auc.dk

${ }^{1}$ J. D. Joannopoulos, R. D. Meade, and J. N. Winn, Photonic Crystals (Princeton University Press, Princeton, NJ, 1995).

${ }^{2}$ Photonic Crystals and Light Localization in the 21st Century, edited by C. M. Soukoulis (Kluwer, Dordrecht, 2001).

${ }^{3}$ T. F. Krauss and R. M. De La Rue, Prog. Quantum Electron. 23, 51 (1999)

${ }^{4}$ T. Baba, N. Fukaya, and J. Yonekura, Electron. Lett. 35, 654 (1999)

${ }^{5}$ S. Yamada, T. Koyama, Y. Katayama, N. Ikeda, Y. Sugimoto, K. Asakawa, N. Kawai, and K. Inoue, J. Appl. Phys. 89, 855 (2001).

${ }^{6}$ M. Lončar, D. Nedeljković, J. Vučković, A. Scherer, and T. P. Pearsall, Appl. Phys. Lett. 77, 1937 (2000).

${ }^{7}$ M. Tokushima, H. Kosaka, A. Tomita, and H. Yamada, Appl. Phys. Lett. 76, 952 (2000).

${ }^{8}$ S. Y. Lin, E. Chow, S. G. Johnson, and J. D. Joannopoulos, Opt. Lett. 25, 1297 (2000)

${ }^{9}$ N. Kawai, K. Inoue, N. Carlsson, N. Ikeda, Y. Sugimoto, K. Asakawa, and T. Takemori, Phys. Rev. Lett. 86, 2289 (2001).

${ }^{10}$ E. Chow, S. Y. Lin, J. R. Wendt, S. G. Johnson, and J. D. Joannopoulos, Opt. Lett. 26, 286 (2001).

${ }^{11}$ A. Talneau, L. Le Gouezigou, and N. Bouadma, Opt. Lett. 26, 1259 (2001).

${ }^{12}$ A. Talneau, L. Le Gouezigou, N. Bouadma, M. Kafesaki, C. M. Soukoulis, and M. Agio, Appl. Phys. Lett. 80, 547 (2002).

${ }^{13}$ M. Lončar, D. Nedeljković, J. Vučković, A. Scherer, S. Kuchinsky, and D. C. Allan, Appl. Phys. Lett. 80, 1689 (2002).
${ }^{14}$ D. P. Tsai, H. E. Jackson, U. Thiel, R. C. Reddick, S. H. Sharp, and R. J. Warmack, Appl. Phys. Lett. 56, 1515 (1990).

${ }^{15}$ A. G. Choo, H. E. Jackson, U. Thiel, G. N. De Brabander, and J. T. Boyd, Appl. Phys. Lett. 65, 947 (1994); H. E. Jackson, S. M. Lindsay, C. D. Poweleit, D. H. Naghski, G. N. De Brabander, and J. T. Boyd, Ultramicroscopy 61, 295 (1995); M. L. M. Balistreri, J. P. Korterik, L. Kuipers, and N. F. van Hulst, Appl. Phys. Lett. 79, 910 (2001).

${ }^{16}$ S. Bourzeix, J. M. Moison, F. Mignard, F. Barthe, A. C. Boccara, C. Licoppe, B. Mersali, M. Allovon, and A. Bruno, Appl. Phys. Lett. 73, 1035 (1998).

${ }^{17}$ G. H. Vander Rhodes, B. B. Goldberg, M. S. Ünlü, S. T. Chu, W. Pan, T. Kaneko, Y. Kokobun, and B. E. Little, Appl. Phys. Lett. 75, 2368 (1999).

${ }^{18}$ A. L. Campillo, J. W. P. Hsu, C. A. White, and C. D. W. Jones, Appl. Phys. Lett. 80, 2239 (2002).

${ }^{19}$ E. B. McDaniel, J. W. P. Hsu, L. S. Goldner, and R. J. Tonucci, Phys. Rev. B 55, 10878 (1997).

${ }^{20}$ P. L. Phillips, J. C. Knight, B. J. Mangan, P. St. J. Russel, M. D. B. Charlton, and G. J. Parker, J. Appl. Phys. 85, 6337 (1999).

${ }^{21}$ S. I. Bozhevolnyi, J. Erland, K. Leosson, P. M. W. Skovgaard, and J. M. Hvam, Phys. Rev. Lett. 86, 3008 (2001).

${ }^{22}$ D. Gérard, L. Berguiga, F. de Fornel, L. Salomon, C. Seassal, X. Letartre, P. Rojo-Romeo, and P. Viktorovitch, Opt. Lett. 27, 173 (2002).

${ }^{23}$ J. Arentoft, T. Søndergaard, M. Kristensen, A. Boltasseva, M. Thorhauge, and L. Frandsen, Electron. Lett. 38, 274 (2002).

${ }^{24}$ DME-DualScope ${ }^{\mathrm{TM}}$, Herlev, Denmark.

${ }^{25}$ T. Søndergaard, A. Bjarklev, J. Arentoft, M. Kristensen, J. 
Erland, J. Broeng, and S. E. B. Libori, Opt. Commun. 194, 341 (2001).

${ }^{26}$ S. I. Bozhevolnyi, B. Vohnsen, and E. A. Bozhevolnaya, Opt. Commun. 172, 171 (1999).

${ }^{27}$ B. Vohnsen and S. I. Bozhevolnyi, J. Microsc. 194, 311 (1999).

${ }^{28}$ M. Totzeck and H. J. Tiziani, Opt. Commun. 138, 365 (1997).

${ }^{29}$ A. Nesci, R. Dändliker, M. Salt, and H.-P. Herzig, Proc. SPIE 4456, 68 (2001).
${ }^{30}$ M. Vaez-Iravani and R. Toledo-Crow, Appl. Phys. Lett. 62, 1044 (1993); S. Pilevar, W. A. Atia, and C. C. Davis, Ultramicroscopy 61, 233 (1995); J. N. Walford, K. A. Nugent, A. Roberts, and R.E. Scholten, Appl. Opt. 38, 3508 (1999); P. L. Phillips, J. C. Knight, J. M. Pottage, G. Kakarantzas, and P. St. J. Russell, Appl. Phys. Lett. 76, 541 (2000); M. L. M. Balistreri, J. P. Korterik, L. Kuipers, and N. F. van Hulst, Phys. Rev. Lett. 85, 294 (2000); A. Nesci, R. Dändliker, and H. P. Herzig, Opt. Lett. 26, 208 (2001). 